\title{
Autoestima de Riesgo y Protección: Una Mediación entre el Clima Familiar y el Consumo de Sustancias en Adolescentes
}

\section{Risk and Protective Self-esteem: A Mediational Role Between Family Environment and Substance Use in Adolescents}

\author{
Teresa I. Jiménez \\ Universidad de Zaragoza, Spain
}

\begin{abstract}
Resumen. El objetivo del presente trabajo es analizar las relaciones directas e indirectas entre la calidad del clima familiar, la autoestima del adolescente considerada desde una perspectiva multidimensional (autoestima familiar, escolar, social y física) y su consumo de sustancias (tabaco, alcohol y marihuana). La muestra está constituida por 414 adolescentes españoles de entre 12 y 17 años estudiantes en centros educativos de secundaria. Para el análisis de los datos se utiliza la técnica estadística de ecuaciones estructurales y se sigue el procedimiento de análisis de efectos mediadores de Holmbeck (1997). Los resultados indican que la autoestima de los adolescentes puede ser considerada desde una doble perspectiva: protectora, en relación con las dimensiones familiar y escolar que muestran una relación negativa con el consumo de sustancias y, de riesgo, en relación con las dimensiones social y física que muestran una relación positiva con dicho consumo. Además, estos dos tipos de autoestima median significativamente la influencia de la calidad del clima familiar en el consumo de sustancias de los adolescentes. Estos resultados se discuten en relación con la literatura previa y se concluye que parece necesario considerar el clima familiar como un antecedente relevante de la autovaloración de los adolescentes y adoptar una perspectiva multidimensional en el estudio de la autoestima de adolescentes consumidores.

Palabras clave: adolescencia, autoestima, clima familiar, consumo de sustancias.
\end{abstract}

\begin{abstract}
The aim of the present study is to analyse the direct and indirect relationships among quality of family environment, multidimensional self-esteem (family, academic, social and physical self-esteem) and substance use (cigarettes, alcohol and marijuana). The study participants were 414 Spanish adolescents aged 12 to 17 years old, drawn from state secondary schools. Statistical analyses were carried out using structural equation modeling and the procedure of mediation effects analysis (Holmbeck, 1997). Results showed a significant mediational effect of self-esteem on the relation between family functioning and adolescent substance use. Moreover, results showed, on the one hand, a protection effect of family and academic self-esteem and, on the other hand, a risk effect of social and physical self-esteem on substance use. Findings are discussed in relation to previous research. As a conclusion, this investigation confirms that family environment is a relevant precedent of adolescent self-evaluation and that it is necessary to adopt a multidimensional perspective when analyse the self-esteem of substance use adolescents.

Keywords: adolescence, family environment, self-esteem, substance use.
\end{abstract}

La adolescencia representa un periodo crítico en el inicio y experimentación en el consumo de sustancias, un tema que siempre ha atraído el interés de los científicos y profesionales de la prevención del consumo de drogas. Por un lado, este interés reside en que en esta población el consumo de sustancias tiene un valor de uso de carácter recreativo asociado a efectos reforzantes para divertirse, evadirse, desinhibirse, relacionarse y experimentar placer (Elzo, 2008), que interfiere significativamente con el adecuado desarrollo psicosocial de los adolescentes. Por otro lado, el interés se convier-

Correspondencia: Teresa I. Jiménez. Departamento de Psicología y Sociología. Facultad de Ciencias Sociales y Humanas. Ciudad Escolar s/n. 44003 Teruel, Spain.E-mail: tijimgut@unizar.es te en preocupación a la vista de los datos de prevalencia de consumo entre los menores de edad: en la población escolar española se observó que durante el mes previo a la encuesta realizada bianualmente por el Observatorio Español sobre Drogas (2009) el 58.5\% había consumido alcohol, el $31.4 \%$ tabaco y el $20.1 \%$ cannabis, unos datos que se han mantenido con algunas variaciones en los últimos años. Además, esta preocupación se agrava por el hecho de que dicho consumo se concentra fundamentalmente en contextos de ocio nocturno y se asocia, en un porcentaje elevado de ocasiones, a otras situaciones de riesgo como conductas de tipo violento (Calafat et al., 2011) y relaciones sexuales no seguras (Prado y Pantin, 2011).

La familia ha sido uno de los contextos en el que muchos investigadores han centrado sus estudios, loca- 
lizando un número importante de factores de riesgo y protección en relación con el consumo de sustancias de los hijos adolescentes. Así, se ha constatado que los adolescentes consumidores perciben a sus familias como contextos marcados por pautas negativas de interacción familiar, donde la comunicación es pobre, los conflictos frecuentes, la cohesión y la flexibilidad bajas, el castigo excesivo y los padres también consumen, observándose que todos estos factores influyen directamente en el consumo de los hijos/as (Butters, 2002; Elzo, 2010; Gilvarry, 2002; Musitu y Pons, 2010; Buelga, Ravenna, Musitu y Lila, 2006). Otros estudios han señalado además que estos factores familiares también pueden influir indirectamente en el consumo de los hijos por tener una relación con otras variables que mantienen una relación estrecha con el consumo de sustancias de los adolescentes (Bhattacharya, 1998; Fagan, Brook, Rubenstone y Zhang, 2005).

En el estudio de estas terceras variables, que podríamos denominar variables mediadoras entre el contexto familiar y la conducta de consumo, la gran mayoría de los trabajos se ha limitado a analizar cómo la calidad de la relación con los padres (conflictiva, poco afectiva y con una comunicación cargada de críticas) puede influir en los procesos de selección de iguales consumidores, en los que su potencial consumo es un factor de riesgo que, a su vez, se relaciona directamente con el consumo de sustancias del hijo o hija adolescente (Bogenschneider, Wu, Raffaelli y Tsay, 1998; Engels, Vitaro, Blokland, de Kemp y Scholte, 2005). Sin embargo es más escasa la investigación de cómo la calidad de la relación familiar toma un significado interno en el adolescente y se refleja en su conducta. Este déficit en la investigación es el que nos ha animado a realizar este estudio en el que pretendemos clarificar el mecanismo a través del cual el clima familiar incide en el consumo de sustancias adolescente, ahondando en la identificación de variables mediadoras que nos ayuden a comprender la interrelación entre estos factores.

Según la formulación más genérica de mediación (Baron y Kenny, 1986), las variables mediadoras explican cómo un acontecimiento o estímulo externo toma un significado psicológico interno influyendo de este modo en las respuestas del sujeto. Desde este punto de vista, las percepciones, actitudes o valores de una persona son potenciales variables mediadoras entre factores ambientales y consecuencias conductuales. En trabajos anteriores hemos observado que la autoestima constituye un recurso psicológico del adolescente que media la relación entre variables familiares y problemas de conducta delictiva (Jiménez, Estévez, Musitu y Murgui, 2007; Jiménez, Lehalle, Murgui y Musitu, 2007). Nos interesa analizar si este tipo de mediación también se observa en relación con el consumo de sustancias en adolescentes. Además, este análisis permite de modo simultáneo examinar el tipo de relación que se establece entre la autoestima, entendida como un constructo multidimensional, y el consumo de sustancias, contribuyendo, de esta manera, a enriquecer la explicación de dicha relación.

Distintos autores señalan que la autoestima es un recurso claro de protección frente a problemas de carácter internalizante como la depresión, donde los adolescentes con menores niveles de autoestima obtienen las mayores puntuaciones en pruebas de sintomatología depresiva (Jiménez, Murgui y Musitu, 2007; McCullough, Huebner y Laughlin, 2000); sin embargo, no está tan claro que sean los adolescentes con más baja autoestima los que se impliquen en mayor medida en problemas externalizantes como la conducta violenta y delictiva y el consumo de sustancias (Butler y Gasson, 2005; Estévez, Martínez y Musitu, 2006). En este sentido, encontramos en la literatura dos tipos de resultados en función del tipo de medida utilizado para evaluar el constructo de autoestima. Por un lado, en los trabajos en los que se utilizan medidas globales se obtienen, generalmente, relaciones de protección, es decir, una relación significativa entre la alta autoestima y el bajo consumo de sustancias (Laure, Binsinger, Ambard y Friser, 2004; Nóbrega, Ferreira, Paredes y Anjos, 2004; Schroeder, Laflin y Weis, 1999). Estos estudios aportan evidencia empírica en la línea general de que una evaluación positiva del sí mismo es un aspecto fundamental para el funcionamiento adaptativo del individuo (Harter, 1990; Taylor y Brown, 1994). Por otro lado, algunos trabajos que utilizan medidas multidimensionales contestan esta visión tradicional de la autoestima, e indican que elevadas puntuaciones en determinados dominios de la autoestima (autoestimas social y física) pueden constituir un potencial factor de riesgo para el desarrollo de problemas de conducta de carácter externalizante (Estévez, Murgui y Musitu, 2009; Musitu y Herrero, 2003; O’Moore y Kirkham, 2001).

Teniendo en cuenta estos antecedentes, el objetivo principal de este estudio es poner a prueba un modelo de mediación donde la autoestima, evaluada desde una perspectiva multidimensional, desempeña un rol mediador entre el clima familiar y el consumo de sustancias del hijo/a adolescente. Para ello seguiremos la formulación de mediación estadística de Baron y Kenny (1986) y el procedimiento propuesto por Holmbeck (1997) para el análisis de efectos mediadores mediante el cálculo de ecuaciones estructurales.

\section{Método}

\section{Participantes}

Participaron en el estudio 414 adolescentes de ambos sexos (el 52\% son chicas) con edades comprendidas entre los 12 y 17 años (edad media 14.2 y desviación típica 1.31), todos ellos estudiantes en centros 
públicos de la comunidad de Castilla y León, ubicados en entornos rurales y urbanos, y distribuidos en $1^{\circ}, 2^{\circ}$, $3^{\circ}$ y $4^{\circ}$ de la Educación Secundaria Obligatoria.

\section{Procedimiento}

Tras un contacto inicial con la dirección de los centros educativos se realizó un seminario con los profesores de cada centro con la finalidad de explicar el interés, objetivos, procedimiento y alcance de la investigación. A través de las Asociaciones de Madres y Padres de Alumnos, se pidió el consentimiento de los padres acerca de la participación de sus hijos/as en el estudio. Los adolescentes participaron de forma voluntaria y anónima en el estudio y cumplimentaron todos los instrumentos en sus aulas correspondientes durante una hora regular de clase. Un investigador previamente entrenado estuvo presente durante todo el proceso de cumplimentación de los instrumentos.

\section{Instrumentos}

Escala de Clima Social Familiar -The Family Environment Scale, FES- de Moos, Moos y Trickett (adaptación al castellano de Fernández-Ballesteros y Sierra, 1984). Esta escala se aplicó para evaluar el funcionamiento de las relaciones personales en la familia a partir de 27 ítems (escala de respuesta verdadero/falso) organizados en tres subescalas: cohesión (por ejemplo, "En mi familia hay un fuerte sentimiento de unión"), expresividad (por ejemplo, "En casa hablamos abiertamente de lo que nos parece o queremos") y conflicto (por ejemplo, "En nuestra familia reñimos mucho"). Los índices de fiabilidad test-retest son $.86, .73$ y .85 respectivamente.

Escala Multidimensional de Autoestima -AF5- de García y Musitu (1999). Esta escala se aplicó para evaluar, a través de 24 ítems (escala de respuesta de 1 -casi nunca- a 5 -casi siempre-), las autopercepciones de los adolescentes en los dominios familiar (por ejemplo, "Me siento querido/a por mis padres"), escolar (por ejemplo, "Soy un/a buen/a estudiante"), social (por ejemplo, "Consigo amigos/as fácilmente") y físico ("Me gusta cómo soy físicamente"). Los índices de fiabilidad según el alpha de Cronbach son $.81, .75, .77$ y .71 respectivamente.

Escala de Consumo de Sustancias de Musitu, Buelga, Lila y Cava (2001). Esta escala evalúa la frecuencia y cantidad de consumo de sustancias tanto legales (tabaco, café y bebidas alcohólicas) como ilegales (derivados del cannabis, cocaína, speed y drogas de diseño). En este trabajo se utilizan los índices referidos al consumo de tabaco (consumo diario de cigarrillos, desde "Nada" a "Más de 25 cigarrillos"), alcohol (consumo semanal, desde "Nada" a "Más de 20 cañas/copas") y marihuana/hachís (consumo semanal, desde "Nada" a "Más de 17 porros") por ser las sustancias más consumidas entre la población adolescente española (Observatorio Español sobre de Drogas, 2006). La fiabilidad test-retest con dos meses de intervalo es de .76 .

\section{Estrategia de análisis de datos}

Se calcularon modelos de ecuaciones estructurales con el programa EQS 6.0 (Bentler, 1995) para analizar la influencia del clima familiar en el consumo de sustancias del adolescente, considerando los distintos tipos de autoestima como variables mediadoras en esta relación. Para examinar estos efectos mediadores, nos guiamos por el procedimiento de análisis para modelos de ecuaciones estructurales sugerido por Holmbeck (1997). También, se realizaron análisis complementarios para conocer la significación y magnitud del posible efecto mediador (Sobel, 1982; McKinnon y Dwyer, 1993). El método de estimación utilizado fue el de Máxima Verosimilitud robusto debido a la desviación de la multinormalidad de los datos (Coeficiente Mardia Normalizado: 32.78). Además, Máxima Verosimilitud es de por sí poco sensible al incumplimiento del supuesto de normalidad multivariada (Hu y Bentler, 1999, West, Finch y Curran, 1995).

Las variables latentes que se incluyeron en los modelos son: (1) Clima Familiar Positivo (indicadores: cohesión y expresividad); (2) Clima Familiar Negativo (indicadores: conflicto); (3) Autoestima-Protección (indicadores: familiar y escolar); (4) AutoestimaRiesgo (indicadores: social y física); (5) Consumo de Sustancias (indicadores: consumo diario de tabaco, consumo semanal de alcohol y consumo semanal de cannabis/hachís).

Para evaluar el ajuste global del modelo se ha calculado el valor del estadístico-ratio de verosimilitud $\chi^{2}$ y el valor de $p$ correspondiente. Los modelos bien ajustados presentan un valor de $\chi^{2}$ no significativos lo que nos viene a indicar que las matrices de entrada previstas y efectivas no son estadísticamente diferentes. Sin embargo, esta medida de calidad del ajuste es muy sensible al tamaño de la muestra de tal modo que si el número de sujetos es elevado (generalmente, a partir de 200), el poder del test aumenta y $\chi^{2}$ alcanza niveles de probabilidad significativos (Hair, Anderson, Tathman y Black, 1999). En consecuencia, es necesario complementar esta medida con otros índices de ajuste tales como el error de aproximación cuadrático medio (RMSEA), el índice de ajuste incremental (IFI), el índice de ajuste comparado (CFI) y el índice de ajuste no-normal de Bentler-Bonnet (NNFI). Los valores iguales o inferiores a .08 para el RMSEA e iguales o superiores a .90 para los IFI, CFI y NNFI, son aceptables e indican un ajuste razonable entre el modelo y los datos (Hair et al., 1999). 


\section{Resultados}

\section{Análisis de correlación preliminares}

Se realizó un análisis de correlación exploratorio entre las variables del estudio que se presenta en la tabla 1 junto con las medias y desviaciones típicas de cada variable. Los resultados indican relaciones significativas entre las variables de cohesión, expresividad y conflicto y la mayoría de las dimensiones de autoestima y de consumo de sustancias. Todas las variables fueron consideradas en las ecuaciones estructurales posteriores.

\section{Modelo de efectos directos}

En relación con el primer requisito señalado por Holmbeck (1997), se calculó un modelo de efectos directos para conocer si el clima familiar predice significativamente el consumo de sustancias de los adolescentes. El modelo directo ajustó bien a los datos: $\chi_{(7,414)}^{2}=8.70(p>.05), \mathrm{CFI}=.99, \mathrm{IFI}=.99, \mathrm{NNFI}=$ .99, y RMSEA $=.02$. Este modelo explicó el $8.9 \%$ de varianza del consumo de sustancias en la muestra. Este modelo y los sucesivos incluyen la correlación entre las variables observables consumo de cannabis y consumo de tabaco. El modelo directo estimado se presen-

Tabla 1. Media, desviación típica y correlaciones entre las variables

\begin{tabular}{|c|c|c|c|c|c|c|c|c|c|c|c|c|}
\hline Variables & Media & Desv.típica & 1 & 2 & 3 & 4 & 5 & 6 & 7 & 8 & 9 & 10 \\
\hline 1. Cohesión & 6.92 & 2.01 & - & & & & & & & & & \\
\hline 2. Expresividad & 5.42 & 1.70 & $.467 * * *$ & - & & & & & & & & \\
\hline 3. Conflicto & 2.79 & 1.72 & $-.424 * * *$ & $-.179 * * *$ & - & & & & & & & \\
\hline 4.Autoestima familiar & 26.58 & 3.59 & $.489 * * *$ & $.379 * * *$ & $-.377 * * *$ & - & & & & & & \\
\hline 5. Autoestima escolar & 21.00 & 4.18 & $.238 * * *$ & $.138 * *$ & $-.127 * *$ & $.373^{* * *}$ & - & & & & & \\
\hline 6. Autoestima social & 24.37 & 3.56 & $.159 * *$ & $.166^{* *}$ & -.015 & $.198 * * *$ & .050 & - & & & & \\
\hline 7. Autoestima física & 20.20 & 4.32 & .054 & .079 & -.007 & .095 & $.192 * * *$ & $.330 * * *$ & - & & & \\
\hline 8. Consumo de tabaco & .81 & .52 & $-.226 * * *$ & -.007 & $.190 * * *$ & $-.307 * * *$ & $-.247 * * *$ & $.110^{*}$ & .028 & - & & \\
\hline 9. Consumo de alcohol & .72 & .46 & $-.155^{* *}$ & -.020 & $.160 * *$ & $-.261 * * *$ & $-.272^{* * *}$ & $.120 *$ & .093 & $.589^{* * *}$ & - & \\
\hline 10. Consumo de cannabis & .56 & .30 & $-.242 * * *$ & -.073 & $.215^{* * *}$ & $-.348 * * *$ & $-.263^{* * *}$ & $.117^{*}$ & .083 & $.679 * * *$ & $.644 * * *$ & - \\
\hline
\end{tabular}

$* * * p<.001 ; * * p<.01 ; * p<.05$

En la tabla 2 se presentan las estimaciones de parámetros no estandarizados, errores estándar y su probabilidad asociada.

Tabla 2. Estimaciones de parámetros no estandarizados, errores estándar y probabilidad asociada

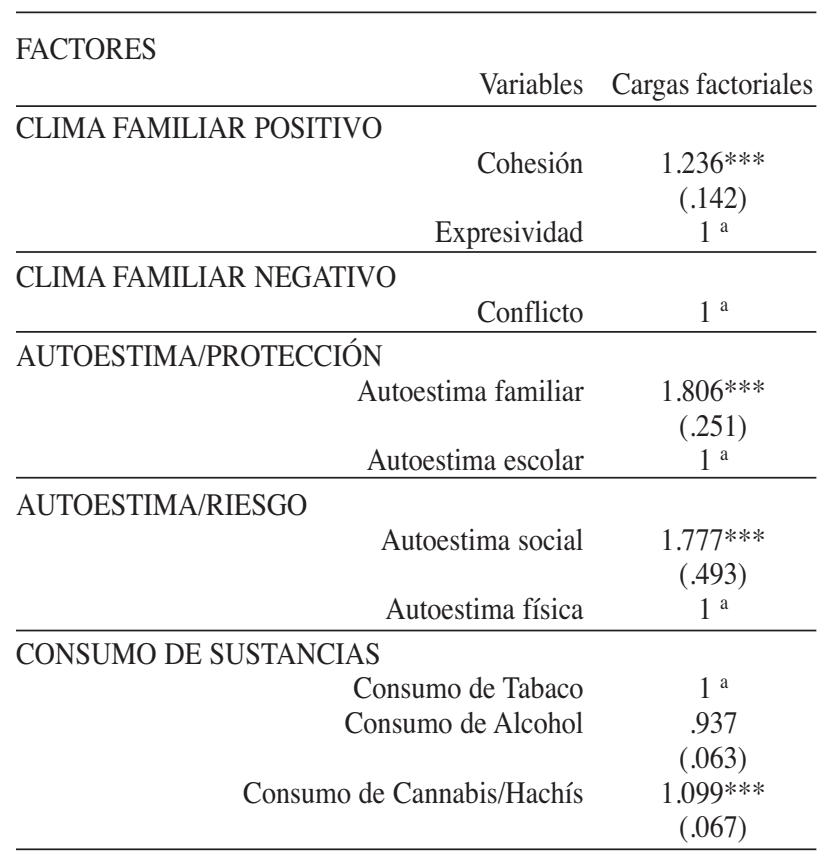

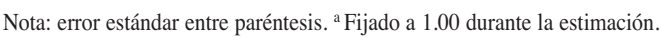
$* * * p<.001$ (bilateral) ta en la figura 1 con los coeficientes estandarizados y su probabilidad asociada.

Figura 1. Modelo estructural directo

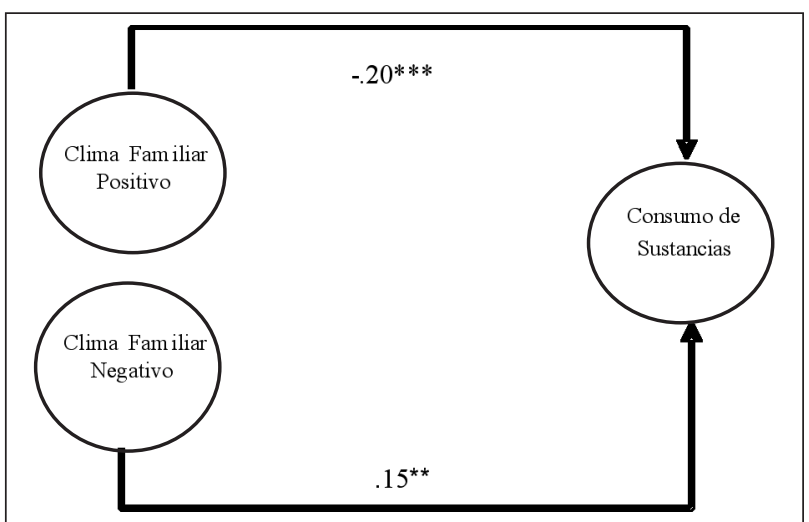

Las líneas continuas representan relaciones significativas entre variables latentes. La significación de las relaciones se ha determinado a partir del error estándar. ${ }^{* *} p<0.001 ; * * p$ $<0.01$

Como se observa en la figura 1 , los coeficientes de relación entre los factores de clima familiar y de consumo de sustancias indican, por un lado, una relación negativa y significativa entre el Clima Familiar Positivo y el Consumo de Sustancias $(\beta=-.20, p<$ .001) y, por otro lado, una relación positiva y significativa entre el Clima Familiar Negativo y el Consumo de Sustancias $(\beta=.15, p<.01)$. 


\section{Modelo de efectos indirectos}

Se calculó un nuevo modelo denominado de efectos indirectos por incluir las relaciones con los factores de autoestima y por tener restringidas a cero las relaciones directas entre los factores de Clima Familiar y de Consumo de Sustancias. El modelo indirecto también ajustó adecuadamente a los datos: $\chi_{(29,414)}^{2}=40.53(p$ $>.05), \mathrm{CFI}=.98, \mathrm{IFI}=.98, \mathrm{NNFI}=.98 \mathrm{y}$ RMSEA $=$ .03. Este modelo explicó el 33\% de varianza del consumo de sustancias en la muestra. Este modelo y los sucesivos incluyen la correlación entre las variables observables autoestima escolar y autoestima familiar. El modelo indirecto estimado se presenta en la figura 2 con los coeficientes estandarizados y su probabilidad asociada.

El examen de los coeficientes de relación entre los factores indica, por un lado, que el factor de Clima
Familiar Positivo se relaciona positiva y significativamente tanto con la Autoestima de Protección $(\beta=.73$, $p<.001)$ como con la Autoestima de Riesgo $(\beta=.32$, $p<.01)$, y que el factor de Clima Familiar Negativo únicamente se relaciona significativamente con la Autoestima de Protección $(\beta=-.21, p<.01)$. Por otro lado, los dos tipos de autoestima presentan relaciones significativas aunque de signo contrario con el Consumo de Sustancias (Autoestima-Protección: $\beta=$ $.55, p<.001$; Autoestima-Riesgo: $\beta=.33, p<.001)$.

\section{Modelo de efectos mediadores}

Se calculó un modelo final denominado de efectos mediadores por incluir tanto las relaciones con los factores mediadores (Autoestima de Riesgo y Protección) como la relación directa entre los factores de clima

Figura 2. Modelo estructural indirecto

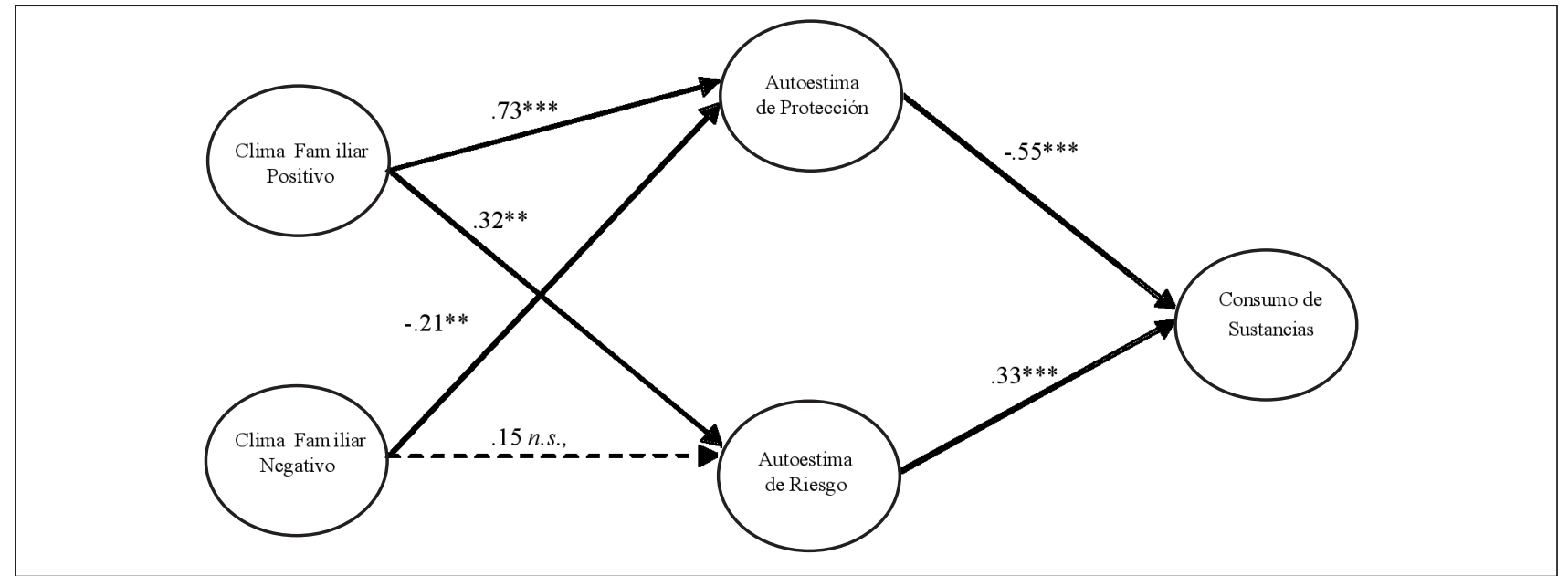

Las líneas continuas representan relaciones significativas entre variables latentes. La significación de las relaciones se ha determinado a partir del error estándar. $* * * p<0.001 ; * * p<0.01$

Figura 3. Modelo estructural mediador

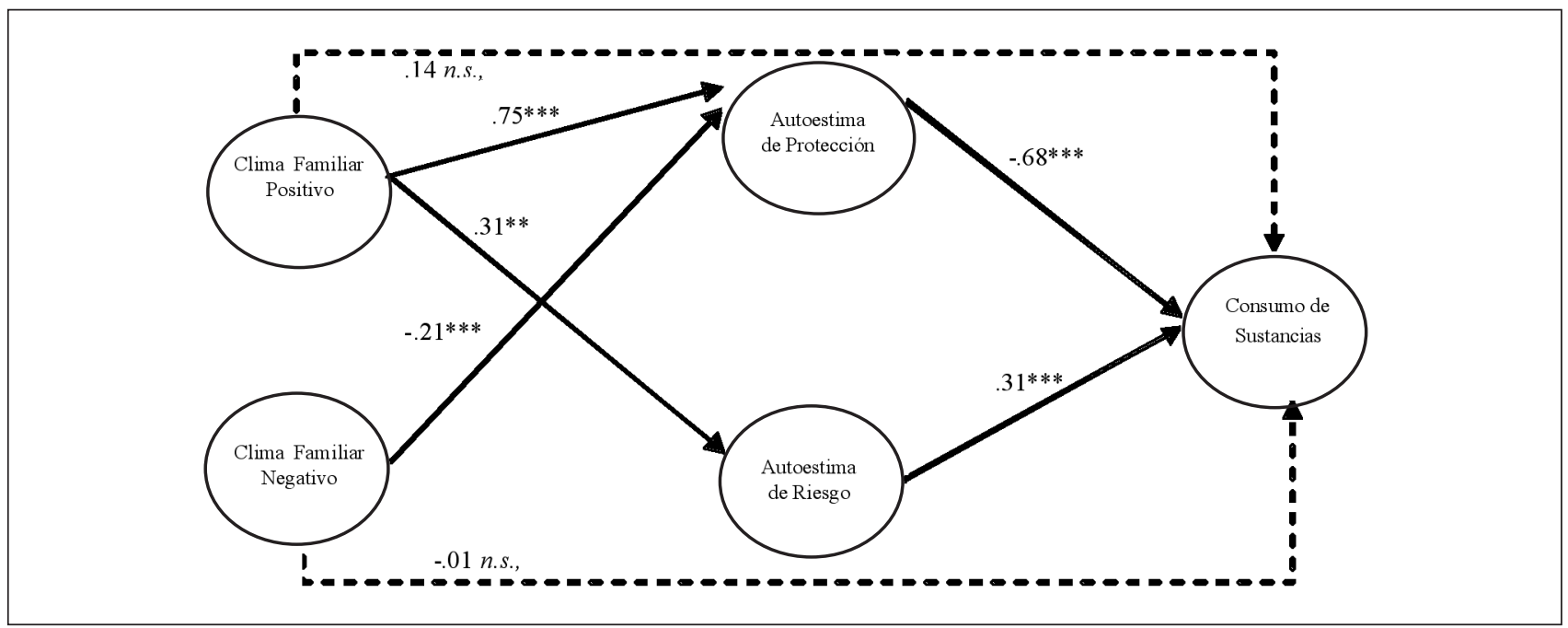

Las líneas continuas representan relaciones significativas entre variables latentes. La significación de las relaciones se ha determinado a partir del error estándar. $* * * p<0.001 ; * * p<0.01$ 
familiar y de consumo de sustancias. Siguiendo el principio de máxima parsimonia (Hair et al., 1999), la relación directa entre Clima Familiar Negativo y Autoestima-Riesgo no se incluyó en el modelo por haberse obtenido en el modelo de efectos indirectos previo que esta relación no era significativa. El modelo mediador ajustó adecuadamente a los datos: $\chi^{2}(27.414)$ $=39.79(p<.01), \mathrm{CFI}=.98, \mathrm{IFI}=.98, \mathrm{NNFI}=.98 \mathrm{y}$ RMSEA = .03. Este modelo explicó el 36,1\% de varianza del consumo de sustancias en la muestra. El modelo mediador estimado se presenta en la figura 3 con los coeficientes estandarizados y su probabilidad asociada.

El examen de los coeficientes de relación entre los factores indica, por un lado, que el factor de Clima Familiar Positivo, de nuevo, se relaciona positiva y significativamente tanto con la Autoestima de Protección $(\beta=.75, p<.001)$ como con la Autoestima de Riesgo $(\beta=.31, p<.01)$, y que, además, el factor de Clima Familiar Negativo se relaciona negativa y significativamente con la Autoestima de Protección $(\beta=-.21, p<.001)$. Sin embargo, las relaciones directas entre los factores de clima familiar y consumo de sustancias, en este caso, han resultado no significativas debido a la presencia de las variables mediadoras de autoestima ( $\beta=.14$, n.s.; $\beta=-.01$, n.s.). En efecto, en este modelo mediador, los dos tipos de autoestima presentan de nuevo relaciones significativas aunque de signo contrario con el Consumo de Sustancias (Autoestima-Protección: $\beta=-.68, p<.001$; Autoestima-Riesgo: $\beta=.31, p<.001$ ).

Para comprobar si este último modelo de mediación aporta o no alguna mejora en el ajuste respecto al modelo indirecto anterior, se compararon ambos modelos mediante el cálculo de la diferencia de los estadísticos $\chi^{2}$. Los resultados de esta prueba resultaron no significativos, indicando que no existen diferencias entre ambos tipos de modelos $\left(\Delta \chi_{(2,414)}^{2}=.74\right)$, para un $\alpha$ de .05 . Esto quiere decir que las relaciones directas entre los factores de clima familiar y de consumo de sustancias añadidas en los modelos mediadores no aportan mejoras significativas en el ajuste respecto del modelo indirecto en el que estas relaciones estaban restringidas.

En la figura 3 referida se observa que, por un lado, tanto el factor Autoestima-Protección como el factor Autoestima-Riesgo son mediadores de la relación entre el Clima Familiar y el Consumo de Sustancias del adolescente. Si atendemos a los signos de los coeficientes de relación observamos que se trata de tres efectos de mediación, dos de ellos con carácter de riesgo: por un lado, el Clima Familiar Positivo potencia las autoevaluaciones positivas del adolescente en todos los dominios de la autoestima, sin embargo los dominios social y físico potencian a su vez el consumo de sustancias del adolescente mientras que los dominios familiar y escolar lo protegen de implicarse en dicho consumo; por otro lado, el Clima Familiar Negativo influye negativa y significativamente en el factor Autoestima-Protección, de tal modo que se incrementa también el riesgo de consumir sustancias.

Finalmente, se realizaron análisis complementarios para conocer la significación de la mediación (test de Sobel, 1982) y el porcentaje del efecto total que es mediado (fórmula de McKinnon, McKinnon y Dwyer, 1993). Los resultados indican que en el modelo mediador calculado, los tres efectos de mediación observados son significativos $(z=2.94, p<.01 ; z=2.40, p<$ .05 y $z=2.56, p<.05)$ y median conjuntamente el $82 \%$ de la relación entre clima familiar positivo y consumo de sustancias.

\section{Discusión}

En el presente estudio se ha analizado el papel mediador de la autoestima en la relación entre el clima familiar y el consumo de sustancias en adolescentes. Los resultados apoyan la función mediadora de la autoestima y sugieren que en dicha relación, las características del funcionamiento familiar constituyen un factor explicativo distal del consumo de sustancias en la adolescencia. Es decir, el clima familiar positivo o negativo potencian o inhiben las autoevaluaciones positivas del adolescente en los distintos dominios relevantes de su vida (familia, escuela, sociabilidad y apariencia física), unas autoevaluaciones que son a su vez importantes predictores del consumo de sustancias de los adolescentes.

Los efectos observados no alcanzan el $100 \%$ de mediación, lo que es indicativo de que en la relación entre clima familiar y consumo de sustancias existen otros potenciales mediadores, si bien este es un resultado habitual en psicología dada la multicausalidad de los problemas objeto de estudio (Baron y Kenny, 1986). Sin embargo, la mediación observada sobrepasa el $80 \%$ en las dos muestras, lo que nos hace pensar que las características de clima familiar, en términos de cohesión, expresividad y conflicto, influyen en el consumo de sustancias de los hijos fundamentalmente porque influyen previamente en sus autoevaluaciones o autoestima.

Estos resultados son coherentes con la perspectiva del Interaccionismo Simbólico según la cual las autoevaluaciones de una persona se construyen a partir de los feedbacks percibidos de los otros significativos (Mead, 1934). En nuestro caso, las percepciones que el adolescente tiene de la calidad de las relaciones en su contexto familiar (el grado de vinculación entre sus miembros, la apertura en la expresividad y el grado de conflicto) influyen en su autoestima y confirman los encontrados por otros autores (Alonso y Román, 2005; Harter, 1990; Musitu et al., 2001).

Respecto de la relación entre la autoestima y el consumo de sustancias, cabe destacar que nuestros resultados apoyan la tesis de que no existe un efecto homogé- 
neo y protector de los distintos tipos de autoestima frente a la implicación del adolescente en problemas de carácter externalizante como el consumo de sustancias. Se confirma que las autoestimas familiar y escolar constituyen una protección frente a este tipo de conductas de riesgo. Sin embargo, los resultados también apoyan la idea de Brendgen y colaboradores (Brendgen, Vitaro, Turgeon, Poulin y Wanner, 2004), según la cual es posible que exista un "lado oscuro" de la autoestima y que una autoestima muy elevada en determinadas dimensiones (social y física) indique un mayor riesgo de implicarse en problemas de carácter externalizante como la conducta disruptiva y el consumo de sustancias. Estos resultados vienen a contestar la imagen tradicional que los investigadores tienen de la autoestima como un recurso psicológico que necesariamente fomenta el ajuste saludable del adolescente (Harter, 1990; Taylor y Brown, 1994) y confirman la necesidad de analizar la autoestima desde una perspectiva multidimensional en trabajos relacionados con el consumo de sustancias en la adolescencia.

En este sentido, es necesario tener en cuenta que el consumo de ciertas sustancias es una conducta fundamentalmente social durante la adolescencia y que el consumo moderado u ocasional es relativamente normativo en el contexto cultural español (Observatorio Español sobre Drogas, 2009). De este modo, es factible pensar que los adolescentes que consumen ciertas sustancias con los iguales (aquellas con un uso más extendido como las evaluadas en este trabajo) se autoperciban como "seres sociales normales" e incluso se autoevalúen positivamente (Musitu y Herrero, 2003). Por tanto, la relación entre consumo y desajuste adolescente también necesita una revisión crítica ya que se comienza a considerar que el consumo de ciertas sustancias, fundamentalmente el alcohol, es un medio que utiliza el adolescente para lograr su ajuste en una sociedad como la nuestra (Pons y Buelga, 2011).

El presente estudio también está sujeto a algunas limitaciones que es necesario señalar. En este sentido, si bien en los modelos mediadores se asume que la variable independiente antecede temporalmente a la mediadora y ésta a la consecuente, no se pueden establecer categóricamente estas relaciones causales entre las variables, dado el carácter transversal de los datos. La disponibilidad de datos en distintos momentos temporales permitiría clarificar el problema de la posible "bidireccionalidad" de los resultados y es una consideración que tenemos presente en próximas investigaciones. Además, sería interesante contrastar estos resultados con estudios que realizasen valoraciones más profundas (por ejemplo, mediante entrevistas en profundidad al sistema familiar) del funcionamiento familiar y del consumo de los adolescente, si bien son evidentes los costes de este tipo de estudio cualitativo con muestras amplias.

En síntesis y pese a las limitaciones señaladas, los resultados de este estudio proveen información impor- tante acerca de los vínculos entre las variables objeto de análisis y presentan una potencial importancia teórica y sugerentes implicaciones prácticas. Parece que, en gran parte, las características del clima familiar influyen en el consumo de sustancias de los hijos/as adolescentes porque influyen en el desarrollo de su autoestima, tanto en la protectora como en la de riesgo. El equilibrio de fuerzas entre estos dos tipos de autoestima del adolescente predice significativamente la implicación de éste en un mayor o menor consumo de sustancias.

En relación con el ámbito de la intervención, sería necesario contemplar la posibilidad de contrarrestar los potenciales efectos negativos de unas autoestimas social y física sobrevaloradas. Un posible camino para este trabajo comenzaría por contestar los modelos e imágenes sociales y culturales que asocian el consumo de determinadas sustancias a una imagen atractiva y sociable de la persona. Además, como se continua señalando con insistencia, las intervenciones preventivas deberían incluir necesariamente al contexto familiar a fin de incrementar su eficacia (Marina, 2010), tanto por tratarse de un referente relacional fundamental de la autovaloración adolescente como por constituir el espacio privilegiado de modelaje de un consumo moderado puesto que, hoy en día, es común entre los profesionales encaminar sus esfuerzos hacia la reducción de daños y de riesgos, más que a la prohibición, que hasta la fecha ha tenido muy poco efecto en el consumo adolescente (Comas, 2007).

\section{Agradecimientos}

Este trabajo ha sido elaborado en el marco del proyecto de investigación PSI2008-01535/PSIC: "Violencia escolar, victimización y reputación social en la adolescencia" subvencionado por el Ministerio de Ciencia e Innovación de España.

\section{Referencias}

Alonso, J. y Román, J. M. (2005). Prácticas educativas familiares y autoestima. Psicothema, 17, 76-82.

Baron, R. M. y Kenny, D. A. (1986). The moderator-mediator variable distinction in social psychological research: conceptual, strategic, and statistical considerations. Journal of Personality and Social Psychology, 51, 11731182.

Bentler, P. M. (1995). EQS Structural Equations Program Manual. Multivariate Software. Encino: CA.

Bhattacharya, G. (1998). Drug use among Asian-Indian adolescents: identifying protective/risk factors. Adolescence, $33,169-184$.

Bogenschneider, K., Wu, M. Y., Raffaelli, M. y Tsay, J. C. (1998). Parent influences on adolescent peer orientation and substance use: The interface of parenting practices 
and values. Child Development, 69, 1672-1688.

Brendgen, M., Vitaro, F., Turgeon, L., Poulin, F. y Wanner, B. (2004). Is there a dark side of positive illusions? Overestimation of social competence and subsequent adjustment in aggressive and nonaggressive children. Journal of Abnormal Child Psychology, 32, 305-320.

Butler, R. J. y Gasson, S. L. (2005). Self esteem/self concept scales for children and adolescents: A review. Child and Adolescent Mental Health, 10, 190-201.

Butters, J. E. (2002). Family stressors and adolescent cannabis use: A pathway to problem use. Journal of Adolescence, 25, 645-654.

Calafat, A., Mantecón, A., Juan, M., Adrover, D., Blay’N., Hughes, K., Bellis, M. y Rosal, F. (2011). Violent behaviour, drunkenness, drug use and social capital in nightlife. Psychosocial Intervention, 20, 45-51.

Comas, D. (2007). Las políticas de juventud en España democrática. Madrid, Instituto de la Juventud.

Elzo, J. (2008). La voz de los adolescentes. Madrid: PPC.

Engels, R. C. M. E., Vitaro, F., Blokland, E. D. E., de Kemp, R. y Scholte y R. H. J. (2004). Influence and selection processes in friendships and adolescent smoking behaviour: The role of parental smoking. Journal of Adolescence, 27, 531-544.

Estévez, E., Martínez, B. y Musitu, G. (2006). La autoestima en adolescentes agresores y víctimas en la escuela: La perspectiva multidimensional. Intervención Psicosocial, 15, 223-232.

Estévez, E., Murgui, S. y Musitu, G. (2009). Psychosocial adjustment in bullies and victims of school violence. European Journal of Psychology of Education, XXIV, 473-483.

Fagan, P., Brook, J. S., Rubenstone, E. y Zhang, Ch. (2005). Parental occupation, education, and smoking as predictors of offspring tobacco use in adulthood: A longitudinal study. Addictive Behaviors, 30, 517-529.

García, F. y Musitu, G. (1999). Autoconcepto Forma 5. Madrid: TEA.

Gilvarry, E. (2002). Substance abuse in young people. Journal of Child Psychology and Psychiatry, 41, 5580.

Harter, S. (1990). Self and identity development. En S. S. Feldman, G. R. Elliot (eds.). At the threshold: The developing adolescent. Cambridge: MA Harvard University Press.

Hair, J. F., Anderson, Jr. R. E., Tathman, R. L. y Black, W. C. (1999). Análisis multivariante. Madrid: Pearson Educación.

Holmbeck, G. N. (1997). Toward terminological, conceptual and statistical clarity in the study of mediators and moderators: Examples from the child-clinical and pediatric psychology literatures. Journal of Consulting and Clinical Psychology, 4, 599-610.

Hu, L. T. y Bentler, P. M. (1999). Cutoff criteria for fit indexes in covariance structure analysis: Conventional criteria versus new alternatives. Structural Equations Modeling, 6, 1-55.

Jiménez, T. I., Estévez, E., Musitu, G. y Murgui, S. (2007).
Comunicación familiar y comportamientos delictivos en la adolescencia: El doble rol mediador de la autoestima. Revista Latinoamericana de Psicología, 39, 473- 485.

Jiménez, T. I., Lehalle, H., Murgui, S. y Musitu, G. (2007). Le rôle de la communication familiale et de l'estime de soi dans la délinquance adolescente. Revue Internationale de Psychologie Sociale, 20, 5-26.

Jiménez, T. I., Murgui, S. y Musitu, G. (2007). Comunicación familiar y ánimo depresivo: el papel mediador de los recursos psicosociales del adolescente. Revista Mexicana de Psicología, 24, 259-271.

Laure, P., Binsinger, C., Ambard, M. F. y Friser, A. (2004). L'intention des pré-adolescents de consommer des substances psychoactives. Les Cahiers Internationnaux de Psychologie Sociale, 62, 89-95.

Marina, J. A. (2010). Programas educativos para la prevención del consumo de alcohol. En J. Elzo, (coord.), Hablemos de alcohol (pp. 93-114). Madrid: Entinema.

McCullough, G., Huebner, E. S. y Laughlin, J. E. (2000). Life events, self concept and adolescents' positive subjective well-being. Psychology in the Schools, 37, 281-290.

McKinnon, D. P. y Dwyer, J. H. (1993). Estimating mediated effects in prevention studies. Evaluation Review, 17, 144-158.

Mead, G. H. (1934). Mind, self and society. Chicago: University of Chicago Press.

Moos, R. H., Moos, B. S. y Trickett, E. J. (1984). Escalas de clima social. Madrid: TEA. (Adaptación española de R. Fernández-Ballesteros y B. Sierra).

Musitu, G., Buelga, S., Lila, M., Cava, M. J. (2001). Familia y adolescencia. Madrid: Síntesis.

Musitu, G. y Pons, J. (2010). Adolescencia y alcohol: Buscando significados en la persona, la familia y la sociedad. En J. Elzo, (coord.), Hablemos de alcohol (pp. 137170). Madrid: Entinema.

Musitu, G. y Herrero, J. (2003). El rol de la autoestima en el consumo moderado de drogas en la adolescencia. Sociotam: Revista Internacional de Ciencias Sociales, 13, 285-306.

Nóbrega, D., Ferreira, A. S., Paredes, T. F. y Anjos, P. A. (2004). Drugs consumption and its relation with self-concept. IX Conference of the European Association for Research on Adolescente. Porto (Portugal).

Observatorio Español sobre Drogas (2009). Encuesta sobre drogas a población escolar. Madrid: Ministerio del Interior. Delegación del Gobierno para el Plan nacional sobre Drogas.

O’Moore, M. y Kirkham, C. (2001). Self-esteem and its relationship to bullying behaviour. Aggressive Behavior, 27, 269-283.

Prado, G. y Pantin, H. (2011). Reducing substance use and HIV health disparities among Hispanic youth in the U.S.A.: The Familias Unidas program of research. Psychosocial Intervention, 20, 63-73.

Pons, J. y Buelga, S. (2011). Factores asociados al consumo juvenil de alcohol: Una revisión desde una perspectiva psicosocial y ecológica. Psychosocial Intervention, 20, 75-94. 
Schroeder, D., Laflin, M. y Weis, D. (1993). The relationship between self.-esteem and drug use: Methodological and statistical limitations of the research. Journal of Drug Issues, 23, 645-665.

Sobel, M. E. (1982). Asymptotic confidence intervals for indirect effects in structural equations models. En S. Leinhardt (ed.), Sociological methodology (pp. 290-312). San Francisco: Jossey-Bass.
Taylor, S. E. y Brown, J. D. (1994). Positive illusions and well-being revisited: Separating fact from fiction. Psychological Bulletin, 116, 21-27.

West, S. G., Finch, J. F. y Curran, P. J. (1995). Structural equations models with non-normal variables. En R. H. Hoyle. Structural equation modeling: Concepts, issues and applications (pp. 56-75). Thousand Oaks, CA, USA: Sage.

Manuscrito Recibido: 16/09/2010

Revisión Recibida: 21/12/2010

Manuscrito Aceptado: 21/12/2010 